\title{
Human Activity Recognition in WSN: A Comparative Study
}

\author{
Muhammad Arshad Awan', Zheng Guangbin ${ }^{1}$, Cheong-Ghil Kim², Shin-Dug Kim ${ }^{1}$ \\ ${ }^{1}$ Department of Computer Science, Yonsei University, 134 Shinchon-dong, Seodaemoon-gu \\ Seoul, 120-749, South Korea. \\ Email:m99arshad@hotmail.com,\{guangbin,sdkim\}@yonsei.ac.kr \\ ${ }^{2}$ Department of Computer Science, Namseoul University \\ Cheonan, South Korea \\ Email:cgkim@nsu.ac.kr
}

\begin{abstract}
Human activity recognition is an emerging field of ubiquitous and pervasive computing. Although recent smartphones have powerful resources, the execution of machine learning algorithms on a large amount of data is still a burden on smartphones. Three major factors including; classification algorithm, data feature, and smartphone position influence the recognition accuracy and time. In this paper, we present a comparative study of six classification algorithms, six data features, and four different positions that are most commonly used in the recognition process using smartphone accelerometer. This analysis can be used to select any specific classification algorithm, data feature, and smartphone position for human activity recognition in terms of accuracy and response time. The methodology we used is composed of two major components; a data collector, and a classifier. A set of eleven activities of daily living, four different positions for data collection and ten volunteers contributed to make it a worth-full comparative study. Results show that K-Nearest Neighbor and J48 algorithms performed well both in terms of time and accuracy irrespective of data features whereas the performance of other algorithms is dependent on the selected data features. Similarly, mean and mode features gave good results in terms of accuracy irrespective of the classification algorithm. A short version of the paper has already been presented at ICIS 2014 .
\end{abstract}

Keywords: Activity recognition; classification algorithm; data feature; smartphone position; ubiquitous computing.

\section{Introduction}

Human activity recognition has become an active field of research in the previous few years due to its direct impact on the life of a common man. Many of the challenges we face in our daily life can be handled by the use of wireless sensors, sensor network, pervasive computing, and artificial intelligence [1]. A number of systems have been developed to recognize human activities in different application areas including; medical, assisted living, sports, and leisure applications [2]. Previously, the human activities were recognized using vision-based approach, and then inertial sensing techniques were being utilized to recognize the human activities. Recently, the smartphones have been used that are equipped with a rich set of sensors, including the accelerometer, GPS, microphone, and etc. The human activity recognition applications that have been developed using smartphone accelerometer are at very naïve state. Different studies used different data features, classification algorithms, and smartphone position, but still there is no optimal solution. The aim of this study is to provide acceptable choices from classification algorithms, feature vectors, and smartphone positions in the activity recognition process. 
The effective use of a smartphone can improve the quality of our life and by using the accelerometer built into the smartphone, one can keep track of his or her activities like walking, jogging, cycling during the day. This information can lead the users to change their habits, e.g., walking or cycling to school instead of taking bus [3]. Human activity recognition process is a combination of signal processing and classification. When a user performs some physical activity like walking or jogging, the horizontal and vertical readings from the accelerometer are recorded. Then a unique set of features is extracted from the raw data, and lastly, some machine learning algorithms are applied for the classification process. Once the known patterns are stored, the application can try to match the new data to the known patterns in order to identify the current activity. The modern smartphones have been equipped with powerful processors and a large amount of memory space, but still it is to overburden them in dealing with signal processing and classification problems. The right choice of the classification algorithm, data feature, and smartphone position is required for improving the accuracy and timely recognition, which is required for different types of applications.

To achieve these motives, a comparative study is carried out. At first, we developed an android application that can be used to get the raw data of accelerometer, while the user is performing an activity. It also labels data, does filtration, extracts the defined features from the raw data and stores it locally in the form of a csv file. A set of eleven basic activities of daily living (ADL) including; laying, sitting, standing, walking, jogging, ascending stairs, descending stairs, ascending elevator, descending elevator, ascending escalator, and descending escalator are selected for experiments. Six classification algorithms that are most commonly used in the process of human activity recognition using smartphone accelerometer including; J48 (decision tree), Naïve Bayes, Bayesian Network, K-Nearest Neighbor, Multilayer Perceptron, and Logistic Regression are selected. Smartphone position to the user's body while performing an activity has a large impact on recognition accuracy. Therefore, four different positions based on the literature survey that give good results are selected, including; hand palm (user is interacting with the phone while performing some physical activity), trousers' pocket (a casual position), armband (user is performing some sports activities), and waist-mounted (easy for elders and patients under care) (Table 1).

Ten volunteers participated in the data collection process. Every user performed all activities, 2 - 3 minutes for each activity, and for each smartphone position. The data filtration is done by removing a few seconds of data at the start and end of each activity. This is required because when the user starts the application to collect data, the smartphone accelerometer starts reading, but the user has to put the cell phone at a specific position and then to perform the activity. Similarly, at the end of an activity, it takes time (few seconds) to take the smartphone and close the application. The sliding-window, a common approach in features extraction, is used for feature extraction with a fixed length window of 2.56 seconds without overlapping. The overlapping is not used to reduce the overhead of mobile module. The window length of 2 - 6 seconds is recommended because very small and very large window lengths have bad impact on recognition accuracy, we are not concerned here on this issue so skipping the detail.

Another factor is the selection of time and/or frequency domain signals depending upon the nature of the

Table 1. List of activities, positions, algorithms and features.

\begin{tabular}{lllll}
\hline Activity & & Position & Algorithm & Feature \\
\hline Laying & Ascending Stairs & Hand Palm (HP) & Decision Tree (J48) & Mean \\
Sitting & Descending Stairs & Trouser Pocket (TP) & Naïve Bayes (NB) & Standard Deviation \\
Standing & Ascending Elevator & Armband (AB) & Bayesian Netwrok (BN) & Correlation \\
Walking & Descending Elevator & Waist Mounted (WM) & K-Nearest Neighbor (KNN) & Variance \\
Jogging & Ascending Escalator & & Multilayer Perceptron (MLP) & Mode \\
& Descending Escalator & & Logistic Regression (LR) & Kurtosis \\
\hline
\end{tabular}

Published by Atlantis Press

Copyright: the authors 
application. Both are used in human activity recognition process using smartphone accelerometer and have their own merits and limitations. We are only taking time domain signals for this study and choose a window length of 2.56 second to keep it in the power of 2, so that we can generate frequency domain signals for our future work. Once the mobile module finishes its task, the collected data is transferred to the workstation for classification and validation process. The extensive classification process is carried out by using six algorithms, each data feature from six features, and each position from four selected positions, making a total of 144 classification models using Weka [4] tool. The detail is explained in methodology and implementation section. 10-fold cross validation, a most commonly used validation method, is used for these experiments.

In a summary, the detail of experiments and results is given in the respective section, the results show that KNearest Neighbor algorithm performed best and J48 also performed well both in terms of time and accuracy irrespective of data features whereas the performances of other algorithms are dependent on the selected data features. The Naïve Bayes algorithm also performed well in terms of time to generate the classification models. Multilayer Perceptron and Logistic Regression performed worst both in terms of time and accuracy. In case of data features, mean and mode features gave good results in terms of accuracy irrespective of the classification algorithm while the recognition accuracy varies for other features based on classification algorithm. It is also observed that a smartphone with tight and fixed position gives more accurate results. The waist-mounted and armband positions gave more accurate results as compared to hand palm (hand held) and trouser pocket positions.

The rest of the paper is organized as follows. Section 2 briefs some related work. The method we used in this study along-with implementation detail is presented in Section 3. The experiments detail and results are discussed in Section 4. Finally, we conclude our paper in the last section.

\section{Related Work}

The activity recognition system can be categorized into two broad categories [5]: video sensor based activity recognition and physical sensor based activity recognition. Physical sensor based activity recognition can be further divided into wearable sensor based activity recognition and object usage based activity recognition. According to this categorization, the embedded sensors of a smartphone can be fitted into wearable sensors' group and similarly the approach may be termed as wearable sensor based activity recognition. Here, we are concerned about this approach.

Various studies have been conducted to recognize human activities in different fields of applications. A methodology [6] was proposed to identify the fall of a patient among other movement activities using 3-axis accelerometer. The postural orientation techniques [7] were used in the study to identify the sit-stand, stand-sit, lie-stand and stand-lie movements, and an accuracy of up-to $84 \%$ was achieved using neural network and knearest neighbor algorithms. Similarly, a study was conducted to distinguish between the fall and activities of daily living (ADL) using tri-axial accelerometer [8].

A number of surveys, including the survey of Lara and Labrador [9] on human activity recognition using wearable sensors have highlighted the importance of selection of data features, classification algorithms and smartphone position in the recognition process. Nevertheless, there is still no optimal solution for the selection of right feature, right algorithm and correct position that can fit into vast areas of applications. Kaghyan et al. [10] reviewed different approaches of human activity recognition, including; external video sensors based, global location tracking and inertial sensors such as accelerometer and highlighted the strengths and weaknesses of each approach. The author emphasized that there is no standard evaluation procedure and human activity recognition using inertial sensors is becoming mature in recent years.

Another recent study [11] analyzed the acceleration signals for the selection of light-weight and accurate features for real-time human activity recognition using smartphones. The authors highlighted the importance of data modeling approach in features extraction process and performed experiments by collecting the data of six different activities to prove the importance of their approach. From these and a work on human activity recognition [12], we got the motivation to have a comparative study, which can cater the choice of the classification algorithm, feature vector, and smartphone position in terms of both time and accuracy. 


\section{Methodology and Implementation}

The proposed method is composed of two major components; one is a mobile module named as data collector, and the other one is workstation module works as a classifier. A brief description and working flow of the proposed methodology is presented in Fig. 1.

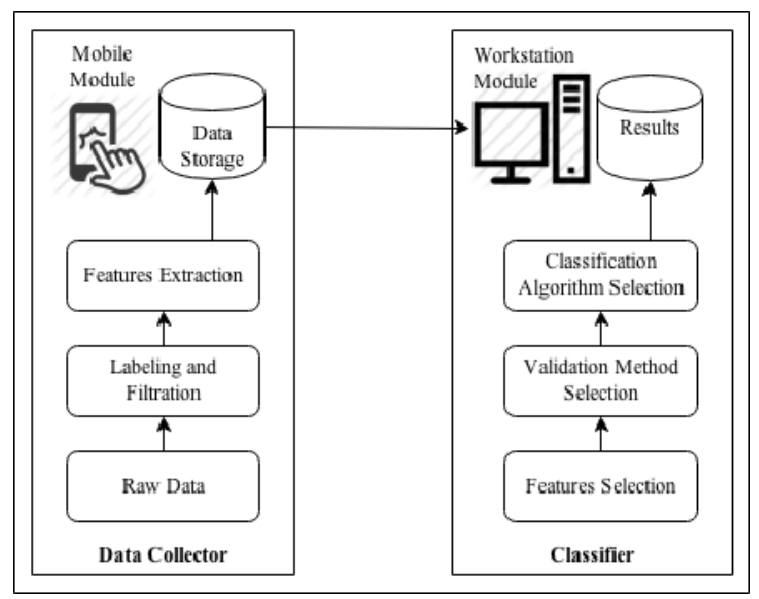

Fig. 1. The proposed methodology

The data collector collects raw data from the accelerometer, labels data, does filtration, and extracts defined features using the sliding-window method. The extracted features in the form of training samples are stored locally. It is a mobile based module and developed as an android application. The user starts the application before starting an activity, selects the smartphone position and an activity from the interface that he or she is going to perform, clicks the start button and puts the cell phone in the selected position and starts doing that activity. After finishing the activity, the stop button is pressed. Feature extraction button is used to extract the defined features, and save button is used to save the training samples in the form of csv files for each selected position. A separate file for each data feature and for each position is saved on the mobile phone.

This procedure is repeated for each activity and every user. The data filtration process is simple and only the removal of a few seconds of raw data at the start and end of each activity because once the user clicks on the start button, the app starts collecting raw data, but the user needs some time to put the phone in the pocket or a designated position and start the activity. Similarly, when the user finishes the activity, it takes some time to take out the smartphone from the pocket and stop the application. A sliding window of 2.56 seconds without overlapping is used for feature extraction as explained in introduction.

Once the training data is collected and saved, it is transferred to the workstation for further classification and validation process. The workstation module selects a training data sample (one feature file at a time), selects the validation method, and applies the selected classification algorithms one by one. The correctly classified instances for each activity and the time taken to build the classification models are saved for comparison. The Weka [4] tool is used on the workstation module for all this procedure and 10-fold cross validation method is used for validation purpose. The position of the smartphone to the user while performing the activity plays an important role alongwith other components in the activity recognition process. The same activity by the same subject cannot be determined if the position of the smartphone does not remain consistent. When considering the literature and real life scenarios, we selected four common positions of the smartphone to the user to overcome the restriction of a particular position while using our application. The user can select the most convenient position of the smartphone depending upon the usage of application. Table 1 shows the Smartphone positions to the user while gathering data for training or recognizing activity. The raw data was labeled according to the selected activity and position by the user from the application interface. A set of 11 activities of daily living (ADL) selected for this study were performed by setting the smartphone in 4 different positions to the user mentioned in Table 1; a total of 44 labeled raw datasets were obtained for the experiments. Table 2 shows the labeling of activities for the 44 raw datasets.

The next task of the data collector was to extract the set of features from the raw data for modeling the classifiers. Feature extraction is a process to apply statistical formulas on the raw data to make it in more refined form. Before features extraction, filtration is necessary to remove the noise from raw data signals. A simple filtration technique is to remove few seconds' raw data from the start and end of an activity data. 
Table 2. List of activities for four different smartphone positions.

\begin{tabular}{llll}
\hline Activity - (HP) & Activity - (TP) & Activity - (WM) & Activity - (AB) \\
\hline Laying-HP & Laying-TP & Laying-WM & Laying-AB \\
Sitting-HP & Sitting-TP & Sitting-WM & Sitting-AB \\
Standing-HP & Standing-TP & Standing-WM & Standing-AB \\
Walking-HP & Walking-TP & Walking-WM & Walking-AB \\
Jogging-HP & Jogging-TP & Jogging-WM & Jogging-AB \\
AscStairs-HP & AscStairs-TP & AscStairs-WM & AscStairs-AB \\
DescStairs-HP & DescStairs-TP & DescStairs-WM & DescStairs-AB \\
AscElevator-HP & AscElevator-TP & AscElevator-WM & AscElevator-AB \\
DescElevator-HP & DescElevator-TP & DescElevator-WM & DescElevator-AB \\
AscEscalator-HP & AscEscalator-TP & AscEscalator-WM & AscEscalator-AB \\
DescEscalator-HP & DescEscalator-TP & DescEscalator-WM & DescEscalator-AB \\
\hline
\end{tabular}

Six data features that are commonly used and provide good results in recognizing human activities by the smartphone accelerometer were chosen based on a literature survey. The list of features is shown in Table 1.

For all the activities, the accelerometer data were collected every 20 milliseconds and 50 samples per second, the normal frequency of the accelerometer embedded in a Smartphone. We collected data for 2.56 seconds, i.e., 128 values for each feature vector of each activity. The selection of 128 values was chosen to maintain the power of 2 to generate the frequency domain signals from time domain signals for future work.

The choice of classification algorithm is debatable based on different factors, especially time and accuracy. Some applications require quick responses, e.g., fall detection in healthcare applications and others require accuracy. We selected the 6 most commonly used machine learning algorithms for detecting human activity using a Ssartphone accelerometer.

The set of eleven activities, smartphone positions, data features, and classification algorithms are presented in Table 1.

A brief procedure from data collection to classification and validation is presented below in bullets to distinguish among the different steps of this process:

- Ten subjects, eleven activities, four positions and approximately 2 - 3 minutes for each activity are selected for these experiments
- Every volunteer performed all activities separately, 2 - 3 minutes for each activity and for each selected position

- A normal frequency of $50 \mathrm{~Hz}$ is selected for smartphone accelerometer

- Data labeling, filtration, and feature extraction is done by android application using the GUI

- $\quad$ The window length of 2.56 seconds is chosen, i.e., 128 values for each feature vector

- A total of approximately 6000 training samples for each feature and for each smartphone position are calculated

- Training data samples for each user, each feature and for each position are stored separately in CSV file format

- Training data samples of all users for each feature and for each position are merged to make a single csv file, e.g., activities-mean-hp.csv (all activities by all users for mean value and for hand palm smartphone position)

- Twenty four training data samples are generated based on each feature and for each position, i.e., $6 \times 4=24$

- Each algorithm is applied to every training data sample one by one

- 144 classification models based on six selected algorithms, six defined features, and four selected positions of smartphone are generated $(6 \times 6 \times 4=$ 144)

- A comparison among algorithms, features, and smartphone positions, i.e., algorithm vs. algorithm, feature vs. feature, position vs. position and their impact on each other in terms of recognition accuracy is conducted 
- Another comparison algorithm vs. algorithm based on selected features is carried out to determine the time taken in the building of a classification model

\section{Experiments and Evaluation}

The extensive work of experiments was carried out on real time data collected through smartphone accelerometer while performing activities with the help of ten volunteers. The comparison among selected algorithms, defined features, and selected smartphone positions (Table 1) was done to recommend the selection of data feature, algorithm and smartphone position in terms of accuracy. A comparison among selected algorithms in terms of time to build classification models was also carried out.

The data in Table 3 and graphs in Fig. 2 \& 3 summarizes the percentage of accuracy based on an algorithm vs. algorithm and feature vs. feature criteria. overall results for algorithm vs. algorithm and features $v s$. feature comparisons are same for each selected position. A separate comparison for recognition accuracy is provided in detail for position vs. position of the smartphone.

It is observed that K-Nearest Neighbor algorithm performed the best and $\mathrm{J} 48$ is in second position in case of any selected feature as can be seen in Table 3 and Fig. 2 \& 3. Whereas Naïve Bayes, Bayesian Network, Multilayer Perceptron, and Logistic Regression algorithms performed well as well as bad depending upon the selected feature. For example, in case of the mean and the mode features, almost all the algorithms performed well as depicted in Fig. 2 \& 3, and for other features, including; standard deviation, correlation, variance, and kurtosis, some algorithms performed well and some bad, which shows the mean and mode features can be considered as the better choice as compared to other selected features. The reason that makes the mean

Table 3. Percentage of accuracy - algorithm vs. algorithm and feature vs. feature (TP position).

\begin{tabular}{lcccccc}
\hline & J48 & NB & BN & KNN & MLP & LR \\
\hline Mean & $99.67 \%$ & $91.27 \%$ & $97.40 \%$ & $100.00 \%$ & $94.17 \%$ & $93.12 \%$ \\
Standard Deviation & $96.86 \%$ & $56.87 \%$ & $77.85 \%$ & $99.60 \%$ & $55.17 \%$ & $58.20 \%$ \\
Correlation & $94.47 \%$ & $52.21 \%$ & $58.74 \%$ & $99.40 \%$ & $55.92 \%$ & $45.93 \%$ \\
Variance & $96.48 \%$ & $55.80 \%$ & $77.85 \%$ & $99.65 \%$ & $47.47 \%$ & $56.97 \%$ \\
Mode & $98.81 \%$ & $76.01 \%$ & $97.13 \%$ & $99.95 \%$ & $81.96 \%$ & $58.29 \%$ \\
Kurtosis & $94.64 \%$ & $47.97 \%$ & $59.61 \%$ & $99.40 \%$ & $50.19 \%$ & $47.40 \%$ \\
\hline
\end{tabular}

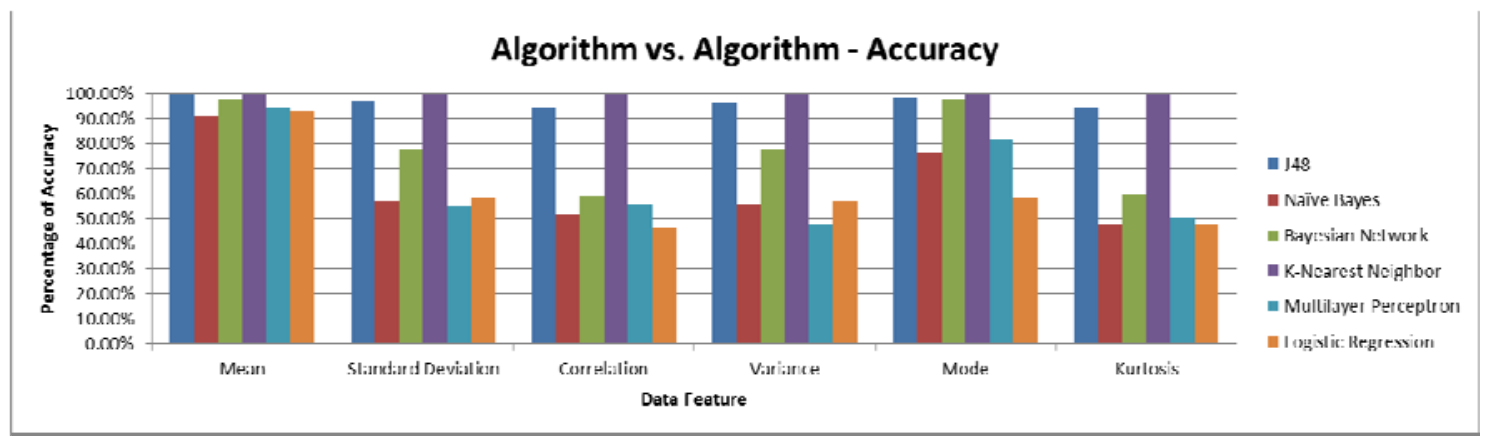

Fig. 2. Algorithm vs. algorithm comparison in terms of accuracy

The results given in the Table 3 are based on the trouser pocket position of the smartphone, which is one of the selected positions. The results from other positions are not shown keeping in view the space constraint, but the and mode features a good choice is that they keep the uniqueness of data patterns (i.e., $\mathrm{x}$-, $\mathrm{y}$-, and $\mathrm{z}$-axis) even in the case of activities that have steady data patterns like laying, sitting, standing, etc. 


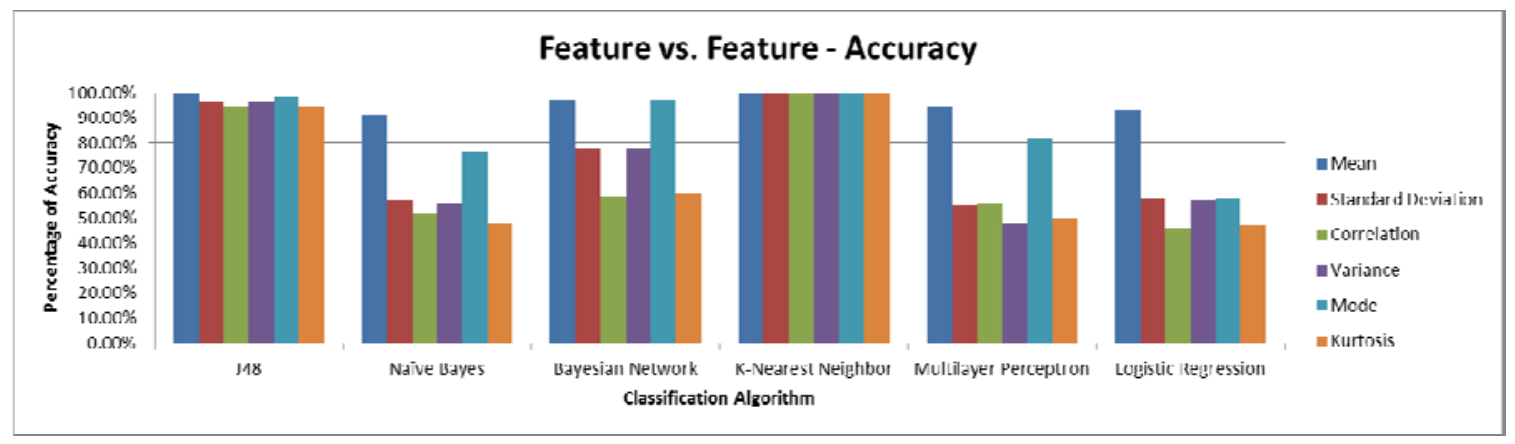

Fig. 3. Feature vs. feature comparison in terms of accuracy

The effect of smartphone position on the recognition accuracy can be depicted from Table 4 and Fig. 4 . The provided comparison is based on the experiments carried out on standard deviation data feature and is almost same for other features if classification algorithm and smartphone position remain consistent. environment, so a little variation may exist in the results, although experiments are performed very carefully.

The waist-mounted (WM) and armband (AB) positions gave more accurate results as compared to handpalm/hand-held (HP) and trouser pocket (TP) positions

Table 4. Percentage of accuracy - position vs. position (standard deviation feature).

\begin{tabular}{lcccccc}
\hline & J48 & NB & BN & KNN & MLP & LR \\
\hline Armband (AB) & $98.40 \%$ & $63.49 \%$ & $81.95 \%$ & $99.79 \%$ & $62.57 \%$ & $67.77 \%$ \\
Hand Palm (HP) & $97.60 \%$ & $63.65 \%$ & $81.38 \%$ & $99.85 \%$ & $63.22 \%$ & $66.97 \%$ \\
Trouser Pocket (TP) & $96.86 \%$ & $56.87 \%$ & $77.85 \%$ & $99.60 \%$ & $55.17 \%$ & $58.20 \%$ \\
Waist Mounted (WM) & $98.11 \%$ & $64.57 \%$ & $86.43 \%$ & $99.86 \%$ & $69.36 \%$ & $72.87 \%$ \\
\hline
\end{tabular}

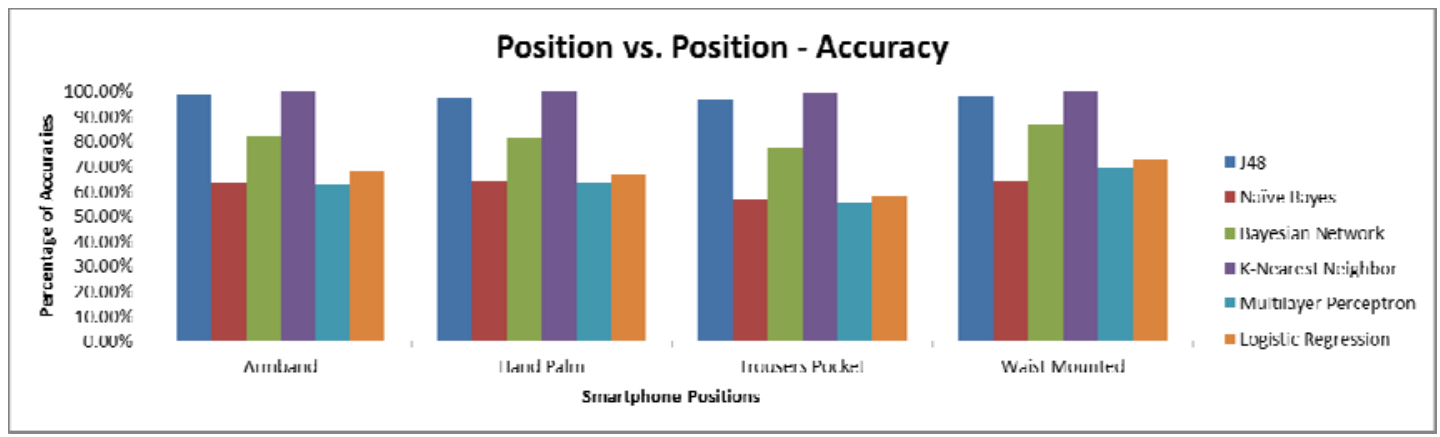

Fig. 4. Position vs. position comparison in terms of accuracy

It is observed that a fixed and tight position of the smartphone to the body of the user can produce more accurate results as compared to unfasten and loose position. It is worth mentioning that these experiments were carried out in an uncontrolled and out of the lab as can be seen in Table 4 and Fig. 4. Although it seems to be a little impact of position on accuracy in some cases, e.g., KNN shows a little variation, but this can increase for a large number of training data samples. 
Similarly, a comparison among selected algorithms in terms of time required to build the classification model is carried out to find a better choice of algorithm for time critical applications. Table 5 and Fig. 5 demonstrate this comparison. It can be seen that KNN and Naïve Bayes took very short time, J48 and Bayes Net took comparatively long time, and Multilayer Perceptron and Logistic Regression took very long time in building classification models.
In case of data features, mean (average) and mode gave good results respectively, in terms of accuracy, irrespective of the classification algorithm, while for other features, recognition accuracy varied based on classification algorithm. The reason was observed that those data features that maintain the uniqueness of data pattern irrespective of their value provide good results. Fig. 7 shows the comparison of data features while keeping the smartphone at waist mounted position and

Table 5. Time taken to build classification model - algorithm vs. algorithm (time in seconds).

\begin{tabular}{lcccccc}
\hline & J48 & NB & BN & KNN & MLP & LR \\
\hline Mean & 0.23 & 0.05 & 0.37 & 0.01 & 17.18 & 7.09 \\
Standard Deviation & 0.19 & 0.01 & 0.06 & 0.01 & 17.62 & 6.44 \\
Correlation & 0.20 & 0.01 & 0.06 & 0.01 & 17.59 & 1.63 \\
Variance & 0.13 & 0.01 & 0.07 & 0.01 & 17.76 & 29.07 \\
Mode & 0.07 & 0.01 & 0.03 & 0.01 & 19.71 & 2.45 \\
Kurtosis & 0.33 & 0.01 & 0.07 & 0.01 & 18.69 & 1.81 \\
\hline
\end{tabular}

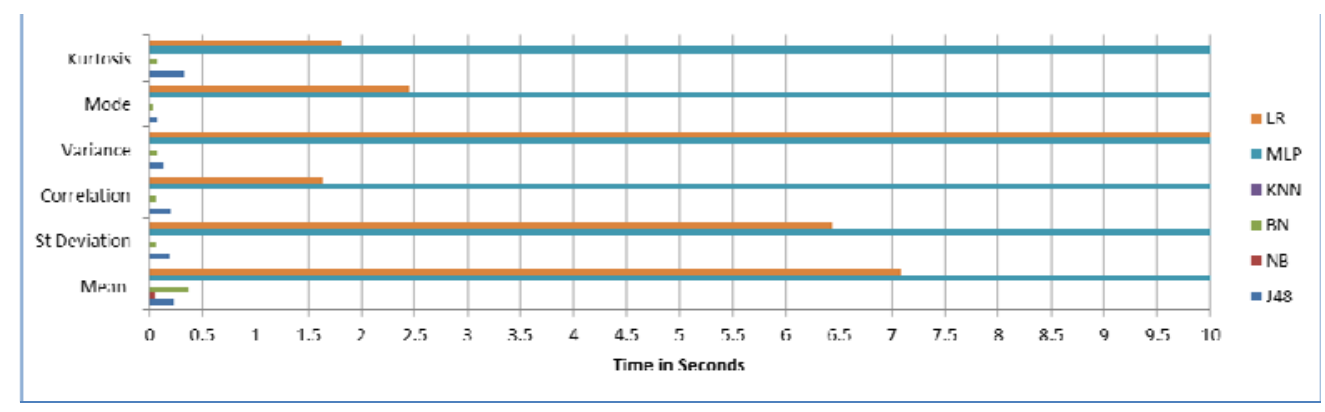

Fig. 5. Time taken to build classification model - algorithm vs. algorithm

In a nutshell, K-Nearest Neighbor algorithm performed best and J48 also performed well both in terms of time and accuracy irrespective of data features, whereas the performances of other algorithms are dependent on the selected data features. Multilayer Perceptron and Logistic Regression algorithms performed worst both in terms of time and accuracy, as can be seen in Fig. 6 as an example. Fig. 6 shows the comparison of classification algorithms while keeping the smartphone at armband position and using the standard deviation as a data feature. Our remarks are based on the experiments' results and not considering the statistical working mechanism of these algorithms. using k-Nearest Neighbor as a classification algorithm. Regarding the smartphone positions, it is also observed that a tight and fixed position of the smartphone can increase the recognition accuracy. The waist-mounted (WM) and armband (AB) positions gave more accurate results as compared to hand palm (HP) and trouser pocket (TP) positions.

The position of the smartphone is also dependent on the type of application, e.g., armband position may be a better choice for a sportsman. Fig. 8 shows the comparison of smartphone positions by using Bayesian Network as a classification algorithm and mean (average) as a data feature. 


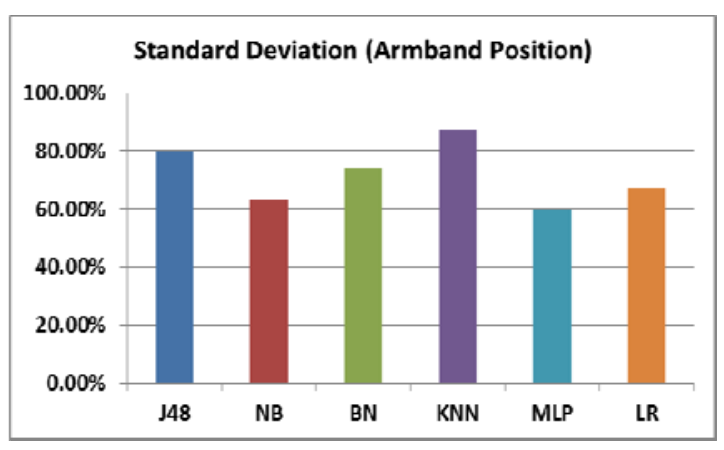

Fig. 6. Comparison of classification algorithms

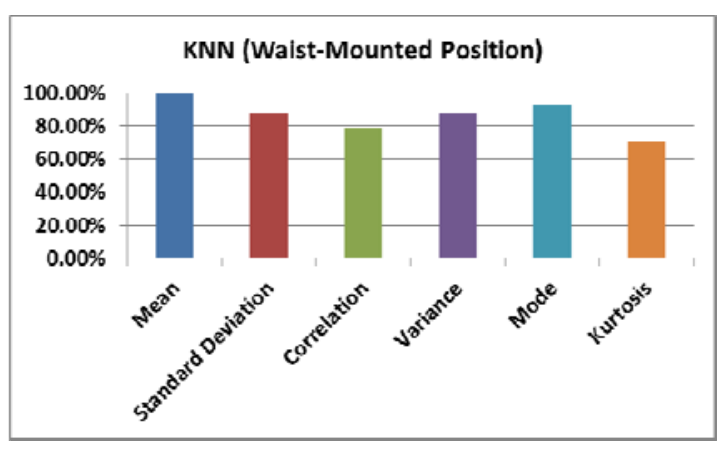

Fig. 7. Comparison of data features

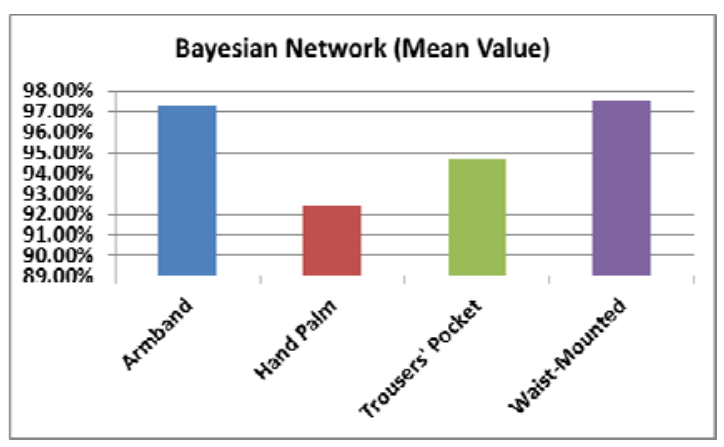

Fig. 8. Comparison of smartphone positions

\section{Conclusion}

This paper demonstrates a comparative study among various algorithms, features and smartphone positions that are commonly used in the process of human activity recognition using smartphone accelerometer. The comparison is based on recognition accuracy and time taken to build classification models. The procedure is carried out by developing an Android application for data collection and then classification and validation process is done on a workstation using Weka tool. A set of eleven activities of daily living, four smartphone positions for data collection and ten volunteers contributed to make it a worth-full comparative study. Results show that K-Nearest Neighbor and J48 algorithms performed well both in terms of time and accuracy irrespective of data features, whereas the performances of other algorithms are dependent on the selected data features. Similarly, mean and mode features gave good results in terms of accuracy irrespective of the classification algorithm. It is also observed that a smartphone with tight and fixed position gives more accurate results. The waist-mounted and armband positions gave more accurate results as compared to hand palm and trousers' pocket positions.

\section{Acknowledgements}

This research was supported by Basic Science Research Program through the National Research Foundation of Korea (NRF) funded by the Ministry of Science, ICT and Future Planning (2012R1A1A2043400). 


\section{References}

1. D. J. Cook, J. C. Augusto and V. R. Jakkula, Ambient intelligence: Technologies, applications, and opportunities, Pervasive and Mobile Computing 5 (2009) 277-298.

2. A. Avci, S. Bosch, M. Marin-Perianu, R. Marin-Perianu and P. Havinga, Activity Recognition Using Inertial Sensing for Healthcare, Wellbeing and Sports Applications: A Survey, in ARCS Workshops (VDE Verlag, 2010), pp. 167-176.

3. L. S. Kmiecik, Cloud Centered, Smartphone Based Longterm Human Activity Recognition Solution, A Project Report, June 2013.

4. WEKA (Waikato Environment for Knowledge Analysis), http://www.cs.waikato.ac.nz/ml/weka/.

5. D. Guan, T. Ma, W. Yuan, Y. K. Lee and A. M. J. Sarkar, Review of Sensor-based Activity Recognition Systems, IETE Technical Review 28 (2011).

6. R. Jafari, W. Li, R. Bajcsy, S. Glaser and S. Sastry, Physical Activity Monitoring for Assisted Living at Home, in BSN, (2007), pp. 213-219.

7. D. M. Karantonis, M. R. Narayanan, M. Mathie, N. H. Lovell and B. G. Celler, Implementation of a Real-Time Human Movement Classifier Using a Tri-axial Accelerometer for Ambulatory Monitoring, IEEE Transactions on Information Technology in Biomedicine 10 (2006) 156-167.

8. A. K. Bourke, J. V. O’Brien and G. M. Lyons, Evaluation of a threshold-based tri-axial accelerometer fall detection algorithm, Gait \& Posture 26 (2007) 194-199.

9. O. D. Lara and M. A. Labrador, A Survey on Human Activity Recognition using Wearable Sensors, IEEE Communications Surveys \& Tutorials 15 (2013) 11921209.

10. S. Kaghyan, H. Sarukhanyan and D. Akopian, Human Movement Classification Approcahes that use Wearbale Sensors and Mobile Devices, SPIE-IS\&T 8667 (2013).

11. A. M. Khan, M. H. Siddiqi and S. W. Lee, Exploratory Data Analysis of Acceleration Signals to Select LightWeight and Accurate Features for Real-Time Activity Recognition on Smartphones, Sensors 13 (2013).

12. M. A. Awan, Z. Guangbin S. D. and Kim, A Dynamic Approach to Recognize Activities in WSN, International Journal of Distributed Sensor Networks 2013 (2013). 